\title{
THE ELECTRO-CAUTERY IN THE TREATMENT OF GLAUCOMA*
}

BY

\author{
Contino Luigi Preziosi, B.Sc., M.D., D.O.(Oxon) \\ MALTA
}

GLAUCOMA is one of the most important diseases in ophthalmic practice considering that preservation of vision, which would otherwise be totally lost, is directly dependent upon a correct diagnosis and proper treatment.

The great benefactor of humanity, Albrecht von Graefe, had discovered in $\mathbf{1 8 5 6}$ that iridectomy could cure glaucoma, and the experience of more than half a century has proved to the ophthalmic world that it is still the operation that should be preferred in cases of congestive glaucoma.

In many cases of simple glaucoma, iridectomy does not reduce the tension to normal, and the disease in these cases continues its course towards complete and irreparable loss of sight. I may add that it has been noticed that in cases with the visual field contracted in some segment almost to the fixation point, loss of central vision supervened soon after this operation.

In this communication I will only deal with the surgical treatment of cases of chronic non-congestive glaucoma, or in other words, of simple glaucoma.

von Graefe had observed that in about six per cent. of cases operated on for glaucoma, a filtering cicatrix followed, and that in about twenty per cent., filtration under the conjunctiva could easily be demonstrated.

de Wecker, having grasped the importance of the filtration of aqueous under the conjunctiva in relation to tension, had suggested that in operating for glaucoma', one should aim at constantly obtaining a filtering cicatrix, and in order to obtain this result, he had suggested the combination of sclerotomy and iridectomy.

Without entering into further details, I may state that in our day most ophthalmic surgeons, in order to cure simple glaucoma by allowing the aqueous to filter under the conjunctiva, perform sclerectomy combined with iridectomy. The two operations preferred, are the sclerecto-iridectomy of Lagrange, brought forward in 1906, and Elliot's trephining, performed for the first time in 1909 ; in the first a small portion of sclera is cut out with the scissors, and in the second a small disc of sclero-cornea with the trephine.

I conceived the idea of doing away with the scissors and the trephine, and establishing the fistula between the anterior chamber

* Read before the Camera Medica of Malta, April 5, 1924. 
and the subconjunctival tissue by means of the electro-cautery; this is the operation that I have the honour to submit to-day to your consideration.

It is many years since I had imagined the possibility of this operation, but I performed it for the first time on January 20,1923 ; since then I have operated on several cases allowing some time to pass between one and the other to study the result, and I have modified somewhat the technique. On February 8, 1924, I operated on the first case, following the procedure I am about to describe, and since then I have operated on three other cases.

After disinfection and cocainization of the eye $I$ inject about ten drops of cocain solution under the conjunctiva. I dissect a wide flap of conjunctiva from the upper scleral segment, and having reached the limbus of the cornea, I pull the flap gently downwards by means of a forceps, and by a few lateral strokes with a Sym's keratome, I split the cornea in two layers for $1.5 \mathrm{~mm}$. or more. Whilst continuing to pull the flap downwards I apply lightly the point of the electro-cautery heated to redness, on the limbus of the cornea, more towards this structure than to the sclera, directing the instrument towards the filtration angle of the anterior chamber. As soon as the cautery has touched the tissues I withdraw it, and I repeat the application several times, until aqueous starts flowing out from the anterior chamber. I replace the conjunctival flap, and fix it with about five stitches, and the operation is over. I use the straight electrode, with the platinum wire about $0.5 \mathrm{~mm}$. in diameter, so that the breadth at its termination is about $1 \mathrm{~mm}$., and the thickness about $0.5 \mathrm{~mm}$.

The advantages I claim to have attained with my operation may be summed up as follows :

1. Simplicity of technique. It cannot be denied that it is much easier to perforate with the electro-cautery than with the trephine : I may add that not infrequently the disc of the trephined portion of sclero-cornea is not found in the aperture left by the trephine, and irrigation of the anterior chamber has to be performed, sometimes for a considerable time, until the disc appears.

2. Impossibility of injuring deep structures. As soon as the aqueous starts flowing out, the electrode which is being only lightly applied to the tissues as already described, loses its redness, and cannot continue to perforate the tissues. In using the trephine it is only natural that a certain amount of pressure has to be used.

3. In those cases where the base of the iris is extensively attached to the posterior surface of the cornea, it is very easy to perforate with the cautery until the aqueous is reached.

4. The anterior chamber is emptied slowly, and consequently the risk of intraocular haemorrhage is smaller. 
5. Absolute asepsis of the instrument that penetrates the eyeball.

6. Iridectomy is not performed.

7. Fragments of iris cannot remain entangled in the sclerocorneal aperture, and consequently the risk of late infection is much smaller.

I now give notes of three patients on whom I have performed my operation :

G.Z. from Zeitun consulted me on December 24 last to have his eyesight tested as he had noticed diminution of vision for some time. This patient was suffering from simple glaucoma in both eyes. Tension of the right eye was 95 , and that of the left eye 85 (McLean). There was in the chart of his visual field of the right exe a paracentral scotoma in continuity with the blind spot, besides the peripheral contraction of the field. Operation was then suggested and refused, and treatment by miotics was therefore prescribed. Later on the patient did not object to the operation, and on February 8 last I operated on his right eye. No atropin drops were instilled in the course of aftertreatment, as the anterior chamber was found to be re-formed at the first dressing, that is on the second day after the operation, and the pupil reacted promptly to light. It is to be noted that the tension is a little below normal, and that the filtration is very marked.

M.C. from Casal Paola. I had examined this patient on September 11, 1923. The vision of the right eye had been totally lost for about a year. The tension of the left eye was +2 , and visual field was contracted almost to the fixation point in its upper segment. The patient had then refused to be operated on, and miotics were therefore prescribed; but lately he objected no longer, and on March 11 I operated on his left eye. In this case the anterior chamber reappeared on the fourth day, and as the pupil was contracted I had atropin instilled in the course of after-treatment. Very marked filtration and normal tension followed the operation.

Sister A. of Casal Lia. I have known this patient since 1914, when I diagnosed simple glaucoma in both eyes with the right eye totally blind, and with marked contraction of the visual field of the left eye mostly at its nasal and upper segment. I had then performed iridectomy on her left eye with very satisfactory results, as up to two years ago the tension had remained normal Since then it started rising again, and miotic drops were prescribed; but lately the tension remained high although the drops were being regularly instilled, and the very small residual field was getting more contracted. On March 14 last I performed my operation on the left eye. No atropin drops were instilled during 
the after-treatment, as at the first dressing, the pupil reacted promptly to light. The filtration is very marked, and the tension is still somewhat subnormal.

The fourth patient, operated on March 11 last, also had normal tension and good filtration.

In concluding, I hope I have rendered easier the operation for simple glaucoma, and as far as I am concerned, I can assure you that facing sufferers from this disease, who usually come to me when they have already lost the sight of one eye, and with a contracted field in the other eye, I feel much easier, and I suggest operation more willingly to these unfortunate sufferers, who without the proper.treatment, would ultimately become totally blind.

\title{
PARALYSIS OF DIVERGENCE IN ENCEPHALITIS LETHARGICA
}

\author{
BY \\ E. R. Chambers \\ BRISTOL
}

THE following case is, I think, worthy of note, as it illustrates an uncommon type of diplopia occurring in the recent epidemic of encephalitis lethargica.

Girl, age 19. Complained of diplopia. Two evenings before seeing me, she had had headache and giddiness and felt sick; on the following morning she awoke with diplopia. The diplopia was very troublesome when looking at distant objects, but was hardly present at all in near vision. There was no drowsiness. Temperature $101^{\circ}$. She had not had influenza nor any other recent illness. Examination of eyes showed no ptosis, no manifest squint, pupils were normal in size, equal, and reacted to light and accommodation. Both optic discs and fundi were normal. The movements of both eyes were normal.

An examination of the diplopia in the six cardinal directions of gaze showed it to be homonymous, with erect images, the unusual feature being that there was no alteration in the relative position of the two images in any of the six cardinal directions of gaze. The first examination was carried out with the candle at three metres from the patient. A second examination, carried out at five metres, showed the two images to be further apart than they were when the examination was performed at three metres from the patient, but, again, their relative positions remained the same in the six cardinal directions of gaze. On approximating the 